University of Denver

Digital Commons @ DU

Mathematics Preprint Series

Mathematics

2012

\title{
A Characterization of Topologically Completely Positive Entropy for Shifts of Finite Type
}

Ronnie Pavlov

Follow this and additional works at: https://digitalcommons.du.edu/math_preprints

Part of the Mathematics Commons

\section{Recommended Citation}

Pavlov, R. (2012). A Characterization of topologically completely positive entropy for shifts of finite type. Mathematics Preprint Series. Retrieved from https://digitalcommons.du.edu/math_preprints/25

This Article is brought to you for free and open access by the Mathematics at Digital Commons @ DU. It has been accepted for inclusion in Mathematics Preprint Series by an authorized administrator of Digital Commons @ DU. For more information, please contact jennifer.cox@du.edu,dig-commons@du.edu. 


\section{A Characterization of Topologically Completely Positive Entropy for Shifts of}

\section{Finite Type}

\section{Comments}

The final version of this article published in the Ergodic Theory and Dynamical Systems is available online at: https://doi.org/10.1017/etds.2013.18 


\title{
A CHARACTERIZATION OF TOPOLOGICALLY COMPLETELY POSITIVE ENTROPY FOR SHIFTS OF FINITE TYPE
}

\author{
RONNIE PAVLOV
}

\begin{abstract}
A topological dynamical system was defined by Blanchard ([1]) to have topologically completely positive entropy (or t.c.p.e.) if its only zero entropy factor is the dynamical system consisting of a single fixed point. For $\mathbb{Z}^{d}$ shifts of finite type, we give a simple condition equivalent to having topologically completely positive entropy. As an application, we use our characterization to derive a similar equivalent condition to t.c.p.e. for the subclass of $\mathbb{Z}^{d}$ group shifts, proved by Boyle and Schraudner in [3]. We also give an example of a $\mathbb{Z}^{2}$ shift of finite type which has topologically completely positive entropy but is not even topologically transitive.
\end{abstract}

\section{INTRODUCTION}

The field of topological dynamics is concerned with the study of topological dynamical systems, where a topological dynamical system is comprised of a compact space $X$ and a group action $\left(T_{g}\right)_{g \in G}$ of self-homeomorphisms of $X$. (In this paper, $G$ will always be $\mathbb{Z}^{d}$ for some $d$.)

Symbolic dynamics is devoted to the study of a specific type of topological dynamical system called a subshift, defined by a finite set $A$ (called an alphabet) and a (possibly infinite) set $\mathcal{F}$ of functions from finite subsets of $\mathbb{Z}^{d}$ to $A$, called patterns. The $\mathbb{Z}^{d}$ subshift $X_{\mathcal{F}}$ induced by $\mathcal{F}$ is then the set of all $x \in A^{\mathbb{Z}^{d}}$ which contain no translate of any of the patterns from $\mathcal{F}$. We always think of $A^{\mathbb{Z}^{d}}$ as being endowed with the (discrete) product topology, and any $\mathbb{Z}^{d}$ subshift as a topological space with the induced topology. Any subshift is a topological dynamical system when acted on by the $\mathbb{Z}^{d}$ shift action of translations by vectors in $\mathbb{Z}^{d}$. A specific type of well-studied subshift occurs when $\mathcal{F}$ is finite. In this case, we call $X_{\mathcal{F}}$ a $\mathbb{Z}^{d}$ shift of finite type, or SFT.

One way to study a topological dynamical system is by way of its factors, or continuous homomorphic images. This motivates Blanchard's definition of topologically completely positive entropy ([1]); a topological dynamical system $\left(X,\left(T_{g}\right)_{g \in G}\right)$ has topological completely positive entropy (or has t.c.p.e.) if it has no zero entropy topological factors aside from the (unavoidable) one-point system endowed with the identity $G$-action. This is an obvious topological analogue of the measuretheoretic notion of a K-system (a measure-theoretical dynamical system is $K$ if it has no zero entropy measure-theoretic factors aside from the identity map on the measure with one-point support), which implies many measure-theoretic mixing

2010 Mathematics Subject Classification. Primary: 37B50; Secondary: 37B10, 37A15.

Key words and phrases. $\mathbb{Z}^{d}$; topological completely positive entropy; shift of finite type; multidimensional. 
properties. However, it was shown in [1] that for $\mathbb{Z}$ subshifts, t.c.p.e. does not imply any topological mixing properties, not even topological transitivity.

The purpose of this paper is to give a simple new condition which is equivalent to t.c.p.e. for $\mathbb{Z}^{d}$ shifts of finite type. For any $\mathbb{Z}^{d}$ SFT $X$, we say that patterns $w, w^{\prime} \in A^{S}$ appearing in points of $X$ are exchangeable in $X$ if there exist points $x, x^{\prime} \in X$ such that $\left.x\right|_{S}=w,\left.x^{\prime}\right|_{S}=w^{\prime}$, and $x$ and $x^{\prime}$ differ on only finitely many sites. Patterns $w, w^{\prime} \in A^{S}$ appearing in points of $X$ are chain exchangeable in $X$ if there exists a finite set of patterns $\left\{w_{i}\right\}_{i=1}^{n}$ such that $w_{1}=w, w_{n}=w^{\prime}$, and $w_{i}$ and $w_{i+1}$ are exchangeable for $1 \leq i<n$.

Theorem 1.1. A $\mathbb{Z}^{d}$ subshift $X$ has topologically completely positive entropy iff

(1) There is a fully supported (shift-invariant probability Borel) measure $\mu$ on $X$.

(2) Any pair of patterns $w, w^{\prime} \in L(X)$ with the same shape are chain exchangeable.

In fact it was already well-known that t.c.p.e. implies (1) for general topological dynamical systems (Corollary 7 from [1]).

Theorem 1.1 unifies some existing proofs in the literature of t.c.p.e. for $\mathbb{Z}^{d}$ SFTs, including the proof of t.c.p.e. in [2] for an example of Hochman ([4]), and a characterization from [3] for the class of $\mathbb{Z}^{d}$ group shifts.

A $\mathbb{Z}^{d}$ subshift $X$ is called a $\mathbb{Z}^{d}$ group shift if the alphabet $A$ of $X$ is a group, and if $X$ is closed under the coordinatewise group operation on $A^{\mathbb{Z}^{d}}$. Group shifts are special examples of $\mathbb{Z}^{d}$ subshifts with many nice properties. For instance, it was noted in [3] (using results from [5]) that any $\mathbb{Z}^{d}$ group shift is a $\mathbb{Z}^{d} \mathrm{SFT}$. In the same paper, they proved (among many other results) the following characterization of t.c.p.e. for group shifts:

Theorem 1.2. ([3], Proposition 2.5, part (4)) If $X$ is a $\mathbb{Z}^{d}$ group shift, then $X$ has t.c.p.e. if and only the homoclinic group

$$
\Delta_{X}:=\left\{x \in X: x(v)=e \text { for all but finitely many } v \in \mathbb{Z}^{d}\right\}
$$

is dense in $X$.

We will show that Theorem 1.2 is a special case of Theorem 1.1.

It was shown in [1] that there exist $\mathbb{Z}$ subshifts which have t.c.p.e. and yet are not even topologically transitive. However, for $\mathbb{Z}$ shifts of finite type, t.c.p.e. implies topological mixing (and even the specification property). Though Theorem 1.1 has somewhat of a topological mixing flavor, we can show that t.c.p.e. also implies no topological mixing properties for $\mathbb{Z}^{d}$ shifts of finite type with $d>1$.

Theorem 1.3. There exists a t.c.p.e. $\mathbb{Z}^{2}$ SFT which is not topologically transitive.

The example from Theorem 1.3 also shows that chain exchangeability in (2) cannot be replaced by the simpler notion of exchangeability:

Theorem 1.4. There exists a t.c.p.e. $\mathbb{Z}^{2}$ SFT which contains a pair of patterns which are not exchangeable.

\section{Definitions AND PRELIMINARIES}

Definition 2.1. For any finite alphabet $A$, the $\mathbb{Z}^{d}$-shift action on $A^{\mathbb{Z}^{d}}$, denoted by $\left\{\sigma_{t}\right\}_{t \in \mathbb{Z}^{d}}$, is defined by $\left(\sigma_{t} x\right)(s)=x(s+t)$ for $s, t \in \mathbb{Z}^{d}$. 
We always think of $A^{\mathbb{Z}^{d}}$ as being endowed with the product discrete topology, with respect to which it is obviously compact.

Definition 2.2. A $\mathbb{Z}^{d}$ subshift is a closed subset of $A^{\mathbb{Z}^{d}}$ which is invariant under the $\mathbb{Z}^{d}$-shift action.

Any subshift inherits a topology from $A^{\mathbb{Z}^{d}}$, and is compact. Each $\sigma_{t}$ is a homeomorphism on any $\mathbb{Z}^{d}$ subshift, and so any $\mathbb{Z}^{d}$ subshift, when paired with the $\mathbb{Z}^{d}$-shift action, is a topological dynamical system.

$\mathbb{Z}^{d}$ subshifts can also be defined in terms of disallowed patterns.

Definition 2.3. A pattern over $A$ is a member of $A^{S}$ for some finite $S \subset \mathbb{Z}^{d}$, which is said to have shape $S$.

For any set $\mathcal{F} \subset A^{*}$, one can define the set $X(\mathcal{F}):=\left\{x \in A^{\mathbb{Z}^{d}}:\left.x\right|_{S} \notin\right.$ $\mathcal{F} \forall$ finite $\left.S \subset \mathbb{Z}^{d}\right\}$. It is well known that any $X(\mathcal{F})$ is a $\mathbb{Z}^{d}$ subshift, and all $\mathbb{Z}^{d}$ subshifts are representable in this way. All $\mathbb{Z}^{d}$ subshifts are assumed to be nonempty in this paper.

For any patterns $v \in A^{S}$ and $w \in A^{T}$ with $S \cap T=\varnothing$, define $v w$ to be the pattern in $A^{S \cup T}$ defined by $\left.(v w)\right|_{S}=v$ and $\left.(v w)\right|_{T}=w$.

Definition 2.4. A $\mathbb{Z}^{d}$ shift of finite type (SFT) is a $\mathbb{Z}^{d}$ subshift equal to $X(\mathcal{F})$ for some finite $\mathcal{F}$. The type of $X$ is defined to be the minimum integer $t$ so that $\mathcal{F}$ can be chosen with all patterns on shapes which are subsets of $[1, t]^{d}$.

(Throughout this paper, for $a<b \in \mathbb{Z},[a, b]$ will be used to denote $\{a, \ldots, b\}$.)

Definition 2.5. The language of a $\mathbb{Z}^{d}$ subshift $X$, denoted by $L(X)$, is the set of all patterns which appear in points of $X$. For any finite $S \subset \mathbb{Z}^{d}, L_{S}(X):=$ $L(X) \cap A^{S}$, the set of patterns in the language of $X$ with shape $S$.

Definition 2.6. For any $\mathbb{Z}^{d}$ subshift $X$ and any finite $S \subseteq \mathbb{Z}^{d}$, patterns $w, w^{\prime} \in$ $L_{S}(X)$ are exchangeable in $X$ if there exists a finite set $T \supseteq S$ and points $x, x^{\prime} \in X$ such that $\left.x\right|_{\mathbb{Z}^{d} \backslash T}=\left.x^{\prime}\right|_{\mathbb{Z}^{d} \backslash T},\left.x\right|_{S}=w$, and $\left.x^{\prime}\right|_{S}=w^{\prime}$.

It should be reasonably clear that if $X$ is an SFT with type $t$, then $w, w^{\prime}$ are exchangeable if and only if there exists $N$ and $v \in L_{[-N, N]^{d} \backslash[-N+t, N-t]^{d}}(X)$ such that $v w, v w^{\prime} \in L(X)$.

Definition 2.7. For any $\mathbb{Z}^{d}$ subshift $X$ and any finite $S \subseteq \mathbb{Z}^{d}$, patterns $w, w^{\prime} \in$ $L_{S}(X)$ are chain exchangeable in $X$ if there exists $n$ and patterns $\left(w_{i}\right)_{i=1}^{n}$ in $L_{S}(X)$ such that $w_{1}=w, w_{n}=w^{\prime}$, and $w_{i}$ and $w_{i+1}$ are exchangeable in $X$ for $1 \leq i<n$.

Alternately, the chain exchangeability relation is just the transitive closure of the exchangeability relation.

Definition 2.8. The topological entropy of a $\mathbb{Z}^{d}$ subshift $X$ is

$$
h(X):=\lim _{n_{1}, \ldots, n_{d} \rightarrow \infty} \frac{1}{\prod_{i=1}^{d} n_{i}} \log \left|L_{\prod_{i=1}^{d}\left[1, n_{i}\right]}(X)\right| .
$$

Definition 2.9. A (topological) factor map is any continuous shift-commuting map $\phi$ from a $\mathbb{Z}^{d}$ subshift $X$ to a $\mathbb{Z}^{d}$ subshift $Y$. 
It is well-known (see [6] for a proof in $d=1$ that extends to arbitrary $d$ ) that any topological factor map $\phi$ between $\mathbb{Z}^{d}$ subshifts is a so-called sliding block code, meaning that there exists a $k$ so that $(\phi(x))(0)$ depends only on $\left.x\right|_{[-k, k]}$. Since $\phi$ is shift-commuting, this also implies that for any rectangular prism $\prod_{i=1}^{d}\left[a_{i}, b_{i}\right]$, $\left.(\phi(x))\right|_{\prod_{i=1}^{d}\left[a_{i}, b_{i}\right]}$ depends only on $\left.x\right|_{\prod_{i=1}^{d}\left[a_{i}-k, b_{i}+k\right]}$. Sometimes we abuse notation and speak of $\phi$ acting on a pattern $w$ with shape $\prod_{i=1}^{d}\left[a_{i}, b_{i}\right]$, in which case the image $\phi(w)$ has shape $\prod_{i=1}^{d}\left[a_{i}-k, b_{i}+k\right]$.

Definition 2.10. A $\mathbb{Z}^{d}$ subshift $X$ has topologically completely positive entropy (or t.c.p.e.) if for every factor map $\phi$ on $X, \phi(X)$ either has positive topological entropy or is a subshift consisting of a single (fixed) point.

We finish with the definition of topological transitivity, a notion of "nondegeneracy" for topological dynamical systems (and in particular, subshifts.)

Definition 2.11. A $\mathbb{Z}^{d}$ subshift $X$ is topologically transitive if for every $w, w^{\prime} \in$ $L(X)$, there exists a point $x \in X$ containing both $w$ and $w^{\prime}$ as subpatterns.

If a subshift is not topologically transitive, then it can be broken into nontrivial pieces which do not interact under the shift map; hence such systems are considered somewhat "degenerate."

We will also have occasion to consider measures on $\mathbb{Z}^{d}$ subshifts; all measures considered in this paper are assumed to be Borel probability measures. This means that they are determined by their values on all cylinder sets, which are sets of the form $[w]:=\left\{x \in X:\left.x\right|_{S}=w\right\}$ for a pattern $w \in L(X)$ with shape $S$.

\section{Proofs}

Proof of Theorem 1.1.

t.c.p.e $\Longrightarrow(1),(2):$ Assume that $X$ is a t.c.p.e. $\mathbb{Z}^{d}$ SFT with type $t$. As stated in the introduction, it is known from [1] that $X$ satisfies (1), but for completeness we give a short self-contained proof here. Suppose for a contradiction that there exists a pattern $w \in L_{S}(X)$ s.t. $\mu([w])=0$ for every measure $\mu$ on $X$. We then claim that for every $\epsilon>0$, there exists $N$ so that for any $n>N$, all patterns of $L_{[-n, n]^{d}}(X)$ contain fewer than $(2 n+1)^{d} \epsilon$ occurrences of $w$. If this were not the case, then there would exist $\epsilon>0$ and a sequence $w_{n} \in L_{[-n, n]^{d}}(X)$ of patterns which each contain at least $(2 n+1)^{d} \epsilon$ occurrences of $w$, and an associated sequence of points $x_{n} \in X$ with $\left.x_{n}\right|_{[-n, n]^{d}}=w_{n}$. Then, the sequence of measures $\frac{1}{(2 n+1)^{d}} \sum_{v \in[-n, n]^{d}} \sigma_{v} \delta_{x_{n}}$ has a weak limit point $\mu$, and it is easy to show that $\mu$ is a shift-invariant measure on $X$ with $\mu([w])>0$.

Define the factor map $\phi: X \rightarrow\{0,1\}^{\mathbb{Z}^{d}}$ by $(\phi(x))(v)=0$ if $\left.x\right|_{v+S} \neq w$ and $(\phi(x))(v)=0$ if $\left.x\right|_{v+S}=w$. Clearly $\phi(X)$ does not consist of a single point. Also, for any $\epsilon>0$, there exists $N$ so that for any $n>N$, all patterns of $L_{[-n, n]^{d}}(\phi(X))$ contain fewer than $(2 n+1)^{d} \epsilon 1 \mathrm{~s}$, meaning that

$h(\phi(X)) \leq \lim _{n \rightarrow \infty} \frac{1}{(2 n+1)^{d}} \log \left(\sum_{i=0}^{\left\lfloor(2 n+1)^{d} \epsilon\right\rfloor}\left(\begin{array}{c}(2 n+1)^{d} \\ i\end{array}\right)\right)=-\epsilon \log \epsilon-(1-\epsilon) \log (1-\epsilon)$.

However, this is true for arbitrary $\epsilon$, and so we would have $h(\phi(X))=0$. As this is a contradiction to t.c.p.e. of $X$, our assumption was wrong, and so for every 
$w \in L(X)$, there exists a measure $\mu_{w}$ on $X$ s.t. $\mu_{w}([w])>0$. If we enumerate all patterns in $L(X)$ as $w_{1}, w_{2}, \ldots$, then clearly the measure $\mu:=\sum_{n=1}^{\infty} 2^{-n} \mu_{w_{n}}$ has full support in $X$, and we have shown that t.c.p.e. of $X$ implies (1).

Now assume for a contradiction that (2) is false, i.e. that there exists finite $S \in \mathbb{Z}^{d}$ and patterns $w, w^{\prime} \in L_{S}(X)$ which are not chain exchangeable. Since chain exchangeability is clearly an equivalence relation, we can partition $L_{S}(X)$ into equivalence classes $C_{1}, \ldots, C_{k}$ for some $k>1$. Define a topological factor map $\psi: X \rightarrow\{1, \ldots, k\}^{\mathbb{Z}^{d}}$ by $(\psi(x))(v)=i$ iff $\left.x\right|_{v+S} \in C_{i}$. We make the following claim: for any $m$, any two patterns $v, v^{\prime} \in L_{[1, m]^{d}}(X)$ which agree on $[1, m]^{d} \backslash[t+1, m-t]^{d}$ have the same image under $\psi$. Suppose for a contradiction that this is not the case, and two such patterns $v, v^{\prime}$ have distinct images $\psi(v) \neq \psi\left(v^{\prime}\right)$. Then there exists $s \in \mathbb{Z}^{d}$ so that $(\psi(v))(s) \neq\left(\psi\left(v^{\prime}\right)\right)(s)$, meaning that $\left.v\right|_{s+S}$ and $\left.v^{\prime}\right|_{s+S}$ lie in distinct classes $C_{i} \neq C_{j}$. However, since $v \in L(X)$, clearly there exists $z \in A^{\mathbb{Z}^{d}} \backslash[1, m]^{d}$ so that $z v \in X$, and since $X$ is an SFT of type $t$ and $v, v^{\prime}$ agree on $[1, m]^{d} \backslash[t+1, m-t]^{d}$, clearly $z v^{\prime} \in X$ as well. Then by definition, $\left.v\right|_{s+S}$ and $\left.v^{\prime}\right|_{s+S}$ are exchangeable, which contradicts the assumption that they lie in different classes. Therefore, our original claim is verified, and $\left.v\right|_{[1, m]^{d} \backslash[t+1, m-t]^{d}}$ uniquely determines $\psi(v)$ for all $m$ and $v \in L_{[1, m]^{d}}(X)$. If $S \subset[-k, k]^{d}$, then this implies that $\left|L_{[1+k, m-k]^{d}}(\psi(X))\right| \leq$ $|A|^{\left|[1, m]^{d} \backslash[t+1, m-t]^{d}\right|} \leq|A|^{2 d t m^{d-1}}$ for all $m$. Then

$$
h(\psi(X))=\lim _{m \rightarrow \infty} \frac{1}{(m-2 k)^{d}} \log \left|L_{[1+k, m-k]^{d}}(\psi(X))\right| \leq \frac{2 d t m^{d-1} \log |A|}{(m-2 k)^{d}}=0 .
$$

Therefore, all pairs $w, w^{\prime} \in L(X)$ with the same shape are chain exchangeable, verifying (2).

(1),(2) $\Longrightarrow$ t.c.p.e.: Suppose that $X$ is a $\mathbb{Z}^{d}$ SFT with type $t$ satisfying (1) and (2), and consider any topological factor map $\phi$ on $X$ for which $\phi(X)$ does not consist of a single (fixed) point. Then there are at least two letters appearing in points of $\phi(X)$, which we can (up to renaming) call $a$ and $b$ without loss of generality. Assume that $m$ is chosen so that $\left.x\right|_{[-m, m]^{d}}$ uniquely determines $(\phi(x))(0)$. There then must exist patterns $w, w^{\prime} \in L_{[-m, m]^{d}}(X)$ so that $\left.x\right|_{[-m, m]^{d}}=w \Longrightarrow(\phi(x))(0)=a$ and $\left.x\right|_{[-m, m]^{d}}=w^{\prime} \Longrightarrow(\phi(x))(0)=b$ for some $a \neq b$ in the alphabet of $\phi(X)$.

By (2), there exist patterns $w_{1}, \ldots, w_{k} \in L_{[-m, m]^{d}}(X)$ so that if we rename $w_{0}:=w$ and $w_{k+1}:=w^{\prime}$, then $w_{i}$ and $w_{i+1}$ are exchangeable for $0 \leq i \leq k$. Clearly there must exist such $i$ so that $\left.x\right|_{[-m, m]^{d}}(X)=w_{i}$ forces $(\phi(x))(0)=c$ and $\left.x\right|_{[-m, m]^{d}}(X)=w_{i+1}$ forces $(\phi(x))(0)=d$ for some letters $c \neq d$ in the alphabet of $\phi(X)$. We rename $v:=w_{i}$ and $v^{\prime}:=w_{i+1}$. Since $v$ and $v^{\prime}$ are exchangeable, there exist $N$ and $z \in A^{\mathbb{Z}^{d} \backslash[-N, N]^{d}}$ so that there are points $x, x^{\prime} \in X$ with $\left.x\right|_{\mathbb{Z}^{d} \backslash[-N, N]^{d}}=$ $\left.x^{\prime}\right|_{\mathbb{Z}^{d} \backslash[-N, N]^{d}}=z,\left.x\right|_{[-m, m]^{d}}=v$, and $\left.x^{\prime}\right|_{[-m, m]^{d}}=v^{\prime}$. Define $u:=\left.x\right|_{[-N-t, N+t]^{d}}$ and $u^{\prime}:=\left.x^{\prime}\right|_{[-N-t, N+t]^{d}}$; then $\left.u\right|_{[-m, m]^{d}}=v,\left.u^{\prime}\right|_{[-m, m]^{d}}=v^{\prime}$, and $u$ and $u^{\prime}$ agree on $[-N-t, N+t]^{d} \backslash[-N, N]^{d}$.

By (1), there exists a measure $\mu$ on $X$ such that $\alpha:=\mu([u])>0$. Therefore, there exists a (generic) point $x \in X$ for which $\left.x\right|_{[-\ell, \ell]^{d}}$ contains at least $\frac{\alpha}{2}(2 \ell+1)^{d}$ occurrences of $u$ for all $\ell$. Since any site is part of at most $(2 N+2 t+1)^{d}$ occurrences of $u$, we may use a simple greedy algorithm to find at least $\frac{\alpha}{2}\left(\frac{2 \ell+1}{2 N+2 t+1}\right)^{d}$ disjoint occurrences of $u$ in any $\left.x\right|_{[-\ell, \ell]}$. For any $\ell$, we may then construct a set $A_{\ell}$ of 
at least $2^{\frac{\alpha}{2}\left(\frac{2 \ell+1}{2 N+2 t+1}\right)^{d}}$ patterns with shape $[-\ell, \ell]^{d}$ by replacing any subset of this set of disjoint occurrences of $u$ by $u^{\prime}$. The resulting patterns are all in $L(X)$ since $\left.x\right|_{[-\ell, \ell]^{d}}$ was in $L(X)$, all replacements involved pairs of patterns which agreed on their boundaries of thickness $t$, and $X$ is an SFT of type $t$.

For any pattern $y \in A_{\ell}, y$ can be completed to at least one point $x \in X$; define $\phi(y)$ to be $\left.\phi(x)\right|_{[-\ell+m, \ell-m]^{d}}$. (This doesn't depend on the choice of $x$ since $y=\left.x\right|_{[-\ell, \ell]^{d}}$ uniquely determines $\left.\phi(x)\right|_{[-\ell+m, \ell-m]^{d}}$.) For any $y \neq y^{\prime} \in A_{\ell}$, there exists $s \in \mathbb{Z}^{d}$ so that $\left.y\right|_{s+[-N-t, N+t]^{d}}=u$ and $\left.y^{\prime}\right|_{s+[-N-t, N+t]^{d}}=u^{\prime}$ or vice versa, implying that $\left.y\right|_{s+S}=v$ and $\left.y^{\prime}\right|_{s+S}=v^{\prime}$ or vice versa. In either case, $(\phi(y))(s) \neq$ $\left(\phi\left(y^{\prime}\right)\right)(s)$, and so $\phi(y) \neq \phi\left(y^{\prime}\right)$. Since any distinct patterns in $A_{\ell}$ have distinct images and since $\phi\left(A_{\ell}\right) \subseteq L_{[-\ell+m, \ell-m]^{d}}(\phi(X))$,

$$
\left|L_{[-\ell+m, \ell-m]^{d}}(\phi(X))\right| \geq\left|\phi\left(A_{\ell}\right)\right| \geq\left|A_{\ell}\right| \geq 2^{\frac{\alpha}{2}\left(\frac{2 \ell+1}{2 N+2 t+1}\right)^{d}},
$$

implying that

$$
h(\phi(X))=\lim _{\ell \rightarrow \infty} \frac{1}{(2 \ell-2 m+1)^{d}} \log \left|L_{[-\ell+m, \ell-m]^{d}}(\phi(X))\right| \geq \frac{\frac{\alpha}{2} \log 2}{(2 N+2 t+1)^{d}}>0 .
$$

Since $\phi$ was arbitrary, we have shown that $X$ has t.c.p.e., completing the proof.

We now verify Theorems 1.3 and 1.4, both of which use the same example.

Proof of Theorems 1.3 and 1.4. We define a $\mathbb{Z}^{2}$ SFT $X$ over the alphabet $A=$ $\{0,1,2\}$. The local rules defining $X$ are the following:

- Any 0 must be adjacent to a 0 either below or to the right of it (but not both), and adjacent to a 0 either above or to the left of it (but not both).

- Any 2 must be adjacent to a 2 either below or to the right of it (but not both), and adjacent to a 2 either above or to the left of it (but not both).

- The patterns ${ }_{0}^{0}, 2,22, \stackrel{0}{0} \underset{0}{0}$, and ${ }_{2}^{2} \underset{2}{2}$ are forbidden.

The reader may check that these rules force the following structure: Any symbol 0 in a point of $X$ is part of an infinite "meandering ribbon" of thickness 1 , which moves diagonally down and to the right, making at least two moves to the right between each move downward. (Informally, the "slope" of any ribbon of 0s is in the interval $[-0.5,0]$.) Also, any symbol 2 in a point of $X$ is part of an infinite "meandering ribbon" of thickness 1 , which moves diagonally down and to the right, making at least two moves downward between each move to the right. (Informally, the "slope" of any ribbon of $2 \mathrm{~s}$ is in the interval $(-\infty,-2]$.) Any point of $X$ consists of one or more of these "ribbons," surrounded by 1s. Though many ribbons of 0s or 2 s can exist within a point of $X$, it is not possible for the two types of ribbons to coexist within the same point of $X$. (See Figure 1.) This means that the symbols 0 and 2 cannot coexist at all within the same point of $X$, and so $X$ is not topologically transitive.

We now claim that the symbols 0 and 2 are also not exchangeable. Consider any $N$ and any point $x \in X$ with $x(0)=0$. Then this central 0 is part of an infinite ribbon, which must intersect $\left.x\right|_{[-N-1, N+1]^{2} \backslash[-N, N]^{2}}$ in two places, one along the left edge and one along the right edge. But then it is impossible for $\left.x\right|_{[-N-1, N+1]^{2} \backslash[-N, N]^{2}}$ to be filled in a way that contains a 2 at the center, since 


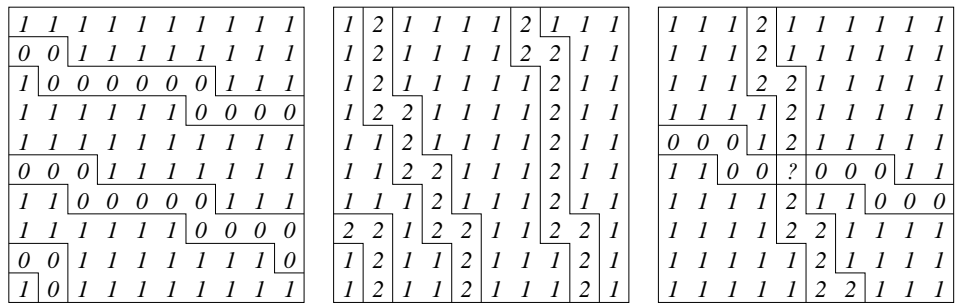

Figure 1. Two patterns in $X$ (and a disallowed pattern with 0 and 2)

the resulting point would contain both a 0 and a 2 , which has already been shown to be impossible. Therefore, 0 and 2 are not exchangeable.

Now, to complete the proof, it suffices to show that $X$ has t.c.p.e, and by Theorem 1.1 it suffices to show that $X$ satisfies (1) and (2). Consider any $m$ and pattern $w \in L_{[-m, m]^{2}}(X)$. Then $w$ consists of pieces of finitely many ribbons (call them $R_{1}, \ldots, R_{j}$ in order from top to bottom) of 0 s surrounded by $1 \mathrm{~s}$, or pieces of finitely many ribbons of $2 \mathrm{~s}$ surrounded by $1 \mathrm{~s}$. We begin with the former case. We will extend $w$ to the left and right, while forcing the ribbons to align into a manageable structure. Since $w \in L(X)$, it can be completed to a point $y \in X$. This point may contain other ribbons of 0 s (besides the $R_{i}$ for $1 \leq i \leq j$ ), but changing them to 1 s will still yield a legal point of $X$ with $w$ occupying $[-m, m]^{2}$, so without loss of generality we can assume that the $R_{i}$ are the only ribbons in $y$. Consider the restriction $\left.y\right|_{[-m, m] \times \mathbb{Z}}$; all $R_{i}$ must hit the left and right edges of the strip $[-m, m] \times \mathbb{Z}$ since ribbons of 0s may not "meander" vertically for more than one consecutive unit. Therefore, there exists $V$ so that $w^{\prime}:=\left.y\right|_{[-m, m] \times[-V, V]}$ contains only the ribbons $R_{i}$ for $1 \leq i \leq j$, each of which intersects the left and right edges of $w^{\prime}$.

Begin with the right edge of $w$. We extend the ribbons $R_{i}$ on the right in the following way: extend the bottom-most ribbon $R_{j}$ to the right by continuing it horizontally. If $R_{j-1}$ and $R_{j}$ were only two units apart on the rightmost edge of $w^{\prime}$, then continue $R_{j-1}$ to the right horizontally; if $R_{j-1}$ was more than two units above $R_{j}$ on the rightmost edge of $w^{\prime}$, then allow $R_{j-1}$ to "meander" down until it is only two units above $R_{j-1}$ (move two units to the right, one unit down, two units to the right, one unit down, etc.), and then continue it horizontally. Then, continue $R_{j-2}$ to the right by making exactly the same movements as $R_{j-1}$ (i.e. just make it a vertical shift of $R_{j-1}$ ) until $R_{j-1}$ is completely horizontal. By the same idea used for $R_{j-1}$, allow $R_{j-2}$ to "meander" (if necessary) until it is exactly two units above $R_{j-1}$, and then continue it horizontally. By continuing with the same idea, we can eventually "align" all of the $R_{i}$ until each consecutive pair is separated vertically by exactly 2 units. We employ a similar idea on the left, until arriving at a rectangular pattern $w^{\prime \prime} \in L_{[-H, H] \times[-V, V]}(X)$ containing $w^{\prime}$ (and therefore $w$ as well), and whose left and right edges each contain $j 0 \mathrm{~s}$, surrounded by $1 \mathrm{~s}$, with a single 1 separating each consecutive pair of 0s. (See Figure 2; in this and all future figures, blank space represents $1 \mathrm{~s}$.) The $j 0$ s on the right are clearly at or below the $j 0$ s on the left; denote their vertical offset by $i$. We can now use $w^{\prime \prime}$ as the fundamental domain of a periodic point $p \in X$ with periods $(2 H+1,-i)$ and $(0,2 \mathrm{~V}+1)$; the period of $(2 H+1,-i)$ simply lines up the $j 0$ s on the right edge of 
any copy of $w^{\prime \prime}$ with the $j$ os on the left edge of an adjoining copy of $w^{\prime \prime}$, continuing the ribbons within $w^{\prime \prime}$ indefinitely, and the period of $(0,2 V+1)$ simply introduces vertical shifts of these ribbons, which clearly do not intersect the originals. (See Figure 3.)

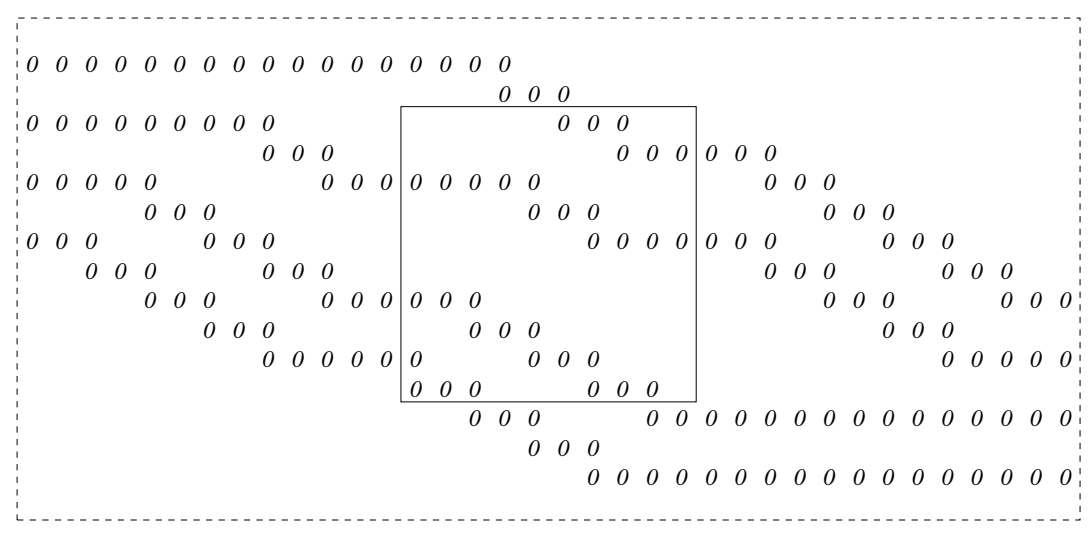

Figure 2. Extending $w$ to $w^{\prime \prime}$

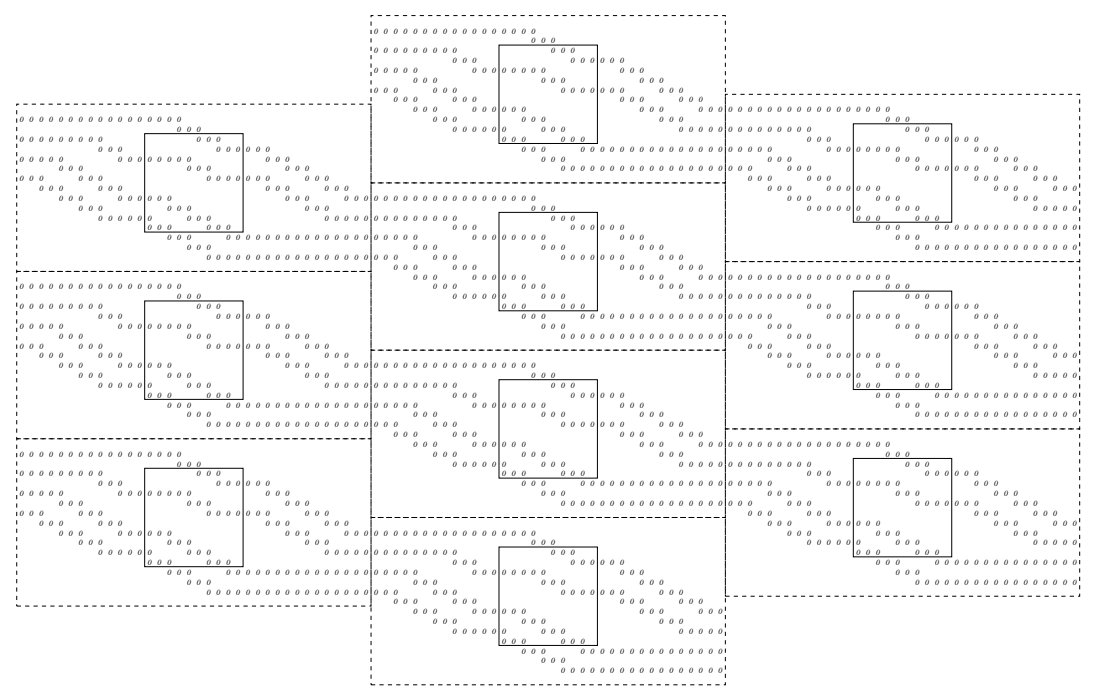

FIGURE 3. Extending $w^{\prime}$ to the doubly periodic point $p$

Since $p$ is doubly periodic, the measure $\mu_{w}:=\frac{1}{(2 H+1)(2 V+1)} \sum_{s \in[-H, H] \times[-V, V]} \delta_{\sigma_{s} p}$ on $X$ is shift-invariant, and since $\left.p\right|_{[-H, H] \times[-V, V]}=w^{\prime \prime}$, clearly $\mu_{w}\left(\left[w^{\prime \prime}\right]\right) \geq \frac{1}{(2 H+1)(2 V+1)}$. Since $w$ is a subpattern of $w^{\prime \prime}, \mu_{w}([w]) \geq \frac{1}{(2 H+1)(2 V+1)}>0$.

If instead $w$ consisted of pieces of finitely many ribbons of $2 \mathrm{~s}$ surrounded by $1 \mathrm{~s}$, we repeat the same proof, with $x$ and $y$-coordinates switched; i.e. all references to "horizontal" are replaced by "vertical" and "left" and "right" are replaced by "top" 
and "bottom" respectively. We have now found, for any $w \in L(X)$ with square shape, a measure $\mu_{w}$ on $X$ with $\mu_{w}([w])>0$. This clearly implies the existence of such a measure for arbitrary $w$, and we may again construct a weighted average $\mu$ of all $\mu_{w}$ which is fully supported in $X$. Therefore, (1) is verified.

We will verify (2) by showing that for any pattern $w \in L_{[-m, m]^{2}}, w$ and the pattern $O_{m}=1^{[-m, m]^{2}}$ of all 1 s with the same shape are exchangeable. Again we begin with the case where $w$ consists of pieces of finitely many ribbons of 0 s surrounded by $1 \mathrm{~s}$, or pieces of finitely many ribbons of $2 \mathrm{~s}$ surrounded by $1 \mathrm{~s}$. By the same technique as in the proof of (1), we may extend $w$ to a pattern $w^{\prime \prime} \in$ $L_{[-H, H] \times[-V, V]}(X)$ whose left and right edges each consist of $j 0 \mathrm{~s}$, surrounded by $1 \mathrm{~s}$, with a single 1 separating each consecutive pair of 0s. By allowing these $j$ ribbons to "meander" simultaneously (i.e. each as a vertical shift of all others), we can extend $w^{\prime}$ to a point $x \in X$ in which the $j$ ribbons become horizontal far enough to the left and right, and in which the vertical offset $i$ between the ribbons in their eventually horizontal state on the left and their eventually horizontal state on the right is at least $2 j+2 m+1$. Choose any $h>2 i+2 j+m$ and any interval $[-v, v]$ which contains all heights of ribbons in $x$. We define two perturbations of $x$, each obtained by deleting $\left.x\right|_{[-h, h] \times[-v, v]}$ and replacing it with another pattern. (See Figure 4.)

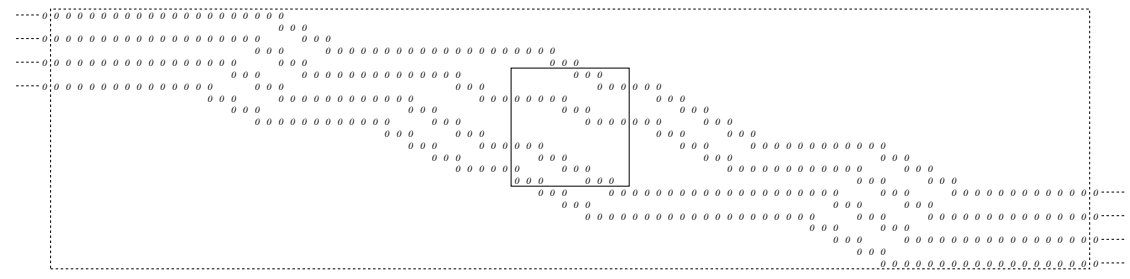

Figure 4. Extending $w$ to $x$

Define $x^{\prime}$ by beginning with the ribbons intersecting the right edge of the hole obtained by deleting $\left.x\right|_{[-h, h] \times[-v, v]}$, and forcing them to "meander" upwards $i$ units (as they move left) as quickly as possible; the top ribbon moves one unit vertically for each horizontal unit, the second ribbon from the top moves horizontally for four units (to avoid becoming connected to the top ribbon) and then begins this pattern, etc. This takes a total horizontal distance of $2 i+2 j$. Then, continue them horizontally to the left until they meet up with the ribbons intersecting the left edge of the hole. Define $x^{\prime \prime}$ by beginning with the ribbons intersecting the left edge of the hole obtained by deleting $\left.x\right|_{[-h, h] \times[-v, v]}$, and forcing them to "meander" downwards $i$ units (as they move right), with two horizontal moves for each vertical move. Then, continue them horizontally to the right until they meet up with the ribbons intersecting the right edge of the hole. Clearly both $x^{\prime}$ and $x^{\prime \prime}$ are in $X$. Since $i \geq 2 j+2 m+1$, the heights of the ribbons on one side of the hole are disjoint from $[-m, m]$; we begin by assuming that it is the right side. Then, consider $x^{\prime}$; recall that the horizontal distance required for "meandering" downwards $i$ units from the left edge is $2 i+2 j$. Since $h>2 i+2 j+m$, in the vertical strip $[-m, m] \times \mathbb{Z}$, the ribbons from the leftt edge have already reached the height of the ones from the right edge, which are disjoint from $[-m, m]$. Therefore, these ribbons do not 
intersect $[-m, m]^{2}$, and so $\left.x^{\prime \prime}\right|_{[-m, m]^{2}}=O_{m}$, i.e. it is filled entirely with 1s. (See Figure 5.) But then $\left.x\right|_{\mathbb{Z}^{2} \backslash[-h, h] \times[-v, v]}$ can be completed to points $x, x^{\prime \prime} \in X$, where $\left.x\right|_{[-m, m]^{2}}=w$ and $\left.x^{\prime \prime}\right|_{[-m, m]^{2}}=O_{m}$, and so $w$ and $O_{m}$ are exchangeable. If instead the heights of the ribbons on the left side of the hole in $x$ were disjoint from $[-m, m]$, we would have used $x^{\prime}$ instead of $x^{\prime \prime}$ and arrived at the same conclusion.

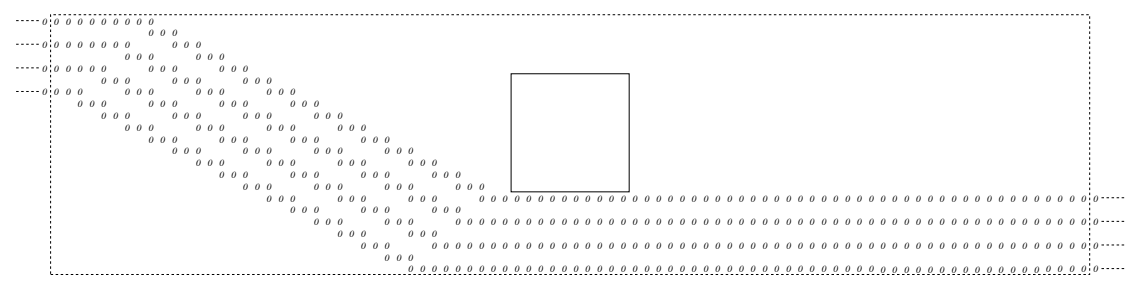

FiguRE 5. Filling hole to make $x^{\prime \prime}$ with no $0 \mathrm{~s}$ in $[-m, m]^{2}$

As before, if instead $w$ consisted of pieces of finitely many ribbons of 2 s surrounded by $1 \mathrm{~s}$, we would repeat the same proof, with $x$ and $y$-coordinates switched. We have then shown that for any $m$, any two patterns of shape $[1, m]^{2}$ are chain exchangeable (by using the pattern $O_{m}$ as the "link.") This clearly implies the same for any pair of patterns with the same (arbitrary) shape, verifying (2) and completing the proof.

We finish by giving two applications of Theorem 1.1. The first is a (re)proof of Theorem 1.2, the characterization of t.c.p.e. for group shifts from [3].

Proof of Theorem 1.2. $\Longrightarrow$ : Assume that $X$ is a $\mathbb{Z}^{d}$ group shift (and therefore a $\mathbb{Z}^{d}$ SFT) for which the homoclinic group $\Delta_{X}$ is not dense. Note that the restriction of $\Delta_{X}$ to any finite set $S$ clearly forms a subgroup of $A^{S}$. So, there exists $S$ for which $G:=\left.\left(\Delta_{X}\right)\right|_{S}$ is a proper subgroup of the group $L_{S}(X)$. Choose any representatives $w, w^{\prime}$ of distinct cosets $C, C^{\prime}$ of $G$ within $L_{S}(X)$. For any $v \in A^{S}$ for which $v$ and $w$ are exchangeable, by definition there exist points $x, x^{\prime} \in X$ so that $\left.x\right|_{S}=v,\left.x^{\prime}\right|_{S}=w$, and $x$ and $x^{\prime}$ differ on only finitely many coordinates. Therefore $x-x^{\prime} \in \Delta_{X}$, meaning that $v-w \in G$, so $v \in C$. Similarly, for any $v^{\prime} \in A^{S}$ for which $v^{\prime}$ and $w^{\prime}$ are exchangeable, $v^{\prime} \in C^{\prime}$. This means that $w$ and $w^{\prime}$ are not chain exchangeable, so (2) is false, and by Theorem 1.1, $X$ does not have t.c.p.e.

$\Longleftarrow$ : Assume that $X$ is a $\mathbb{Z}^{d}$ group shift (and therefore a $\mathbb{Z}^{d}$ SFT) for which the homoclinic group $\Delta_{X}$ is dense. Then for any $w, w^{\prime} \in L_{S}(X)$, there exists a point $d \in \Delta_{X}$ with $\left.d\right|_{S}=w^{\prime}-w$. Since $w \in L(X)$, there exists $x \in X$ with $\left.x\right|_{S}=w$. Then $x+d \in X$ as well, and clearly $\left.(x+d)\right|_{S}=w+\left(w^{\prime}-w\right)=w^{\prime}$. Since $d \in \Delta_{X}$, $x$ and $x+d$ differ on only finitely many coordinates, and so by definition $w$ and $w^{\prime}$ are exchangeable. Since $w$ and $w^{\prime}$ were arbitrary, we have verified (2).

To verify (1), first note that since there are only finitely many patterns in $L_{S}(X)$, and since $\Delta_{X}$ is dense, there exists $N$ so that for any $u \in L_{S}(X)$, there is a point $x^{(u)} \in X$ for which $\left.x^{(u)}\right|_{S}=u$ and $\left.x^{(u)}\right|_{\mathbb{Z}^{d} \backslash[1, N]^{d}}$ consists entirely of identity symbols. Now, consider any $w \in L_{S}(X)$ and any $x \in X$. For any $v \in \mathbb{Z}^{d}$, consider the 
pattern $p(v):=\left.x\right|_{N v+S}$. We can change $p(v)$ to $w$ by simply adding $\sigma_{N v} x^{(w-p(v))}$ to $x ;\left.x^{(w-p(v))}\right|_{S}=w-p(v)$, so $\left.\left(\sigma_{N v} x^{(w-p(v))}\right)\right|_{N v+s}=w-p(v)$. Also, this addition does not change any letters of $x$ outside $\left.x\right|_{N v+[1, N]^{d}}$. We now define a new element of $X$ :

$$
y:=x+\sum_{v \in \mathbb{Z}^{d}} \sigma_{N v} x^{(w-p(v))} .
$$

Since all cubes $N v+[1, N]^{d}$ are disjoint, for every $s \in \mathbb{Z}^{d}$, the letter at location $s$ is the identity for all but at most one term of this sum. Therefore, each change from $p(v)$ to $w$ for the pattern at location $N v+S$ persists in $y$, and so $\left.y\right|_{N v+S}=w$ for all $v \in \mathbb{Z}^{d}$. We now define a measure $\mu_{w}$ as any weak limit point (as $m \rightarrow \infty$ ) of the sequence $\frac{1}{(2 m+1)^{d}} \sum_{v \in[-m, m]^{d}} \sigma_{v} \delta_{y}$; clearly $\mu_{w}$ is shift-invariant and $\mu_{w}([w]) \geq$ $\frac{1}{N^{d}}>0$. As before, we can take a weighted average of the $\mu_{w}$ to define a shiftinvariant measure $\mu$ fully supported in $X$, verifying (1).

We have shown that $X$ satisfies (1) and (2), and so by Theorem 1.1, $X$ has t.c.p.e.

Our second application is very simple, but surprising. We first need definitions of two uniform topological mixing properties for $\mathbb{Z}^{d}$ subshifts.

Definition 3.1. A $\mathbb{Z}^{d}$ subshift $X$

(1) is called block gluing if there exists $g>0$ such that for any pair of two rectangular prisms $R_{1}, R_{2}$ with distance $d\left(R_{1}, R_{2}\right):=\min _{r_{1} \in R_{1}, r_{2} \in R_{2}} d\left(r_{1}, r_{2}\right)>$ $g$ and any pair of points $y, z \in X$, there exists a point $x \in X$ such that $\left.x\right|_{R_{1}}=\left.y\right|_{R_{1}}$ and $\left.x\right|_{R_{2}}=\left.z\right|_{R_{2}}$.

(2) has the uniform filling property (UFP) if there exists $l>0$ such that for any rectangular prism $R=\prod_{i=1}^{d}\left[a_{i}, b_{i}\right]$ and any pair of points $y, z \in$ $X$, there exists a point $x \in X$ with $\left.x\right|_{R}=\left.y\right|_{R}$ and $\left.x\right|_{\mathbb{Z}^{d} \backslash \prod_{i=1}^{d}\left[a_{i}-l, b_{i}+l\right]}=$ $\left.z\right|_{\mathbb{Z}^{d} \backslash \prod_{i=1}^{d}\left[a_{i}-l, b_{i}+l\right]} \cdot$

It was shown in [2] (using techniques already present in [8], for instance) that any block gluing $\mathbb{Z}^{d}$ SFT has topologically completely positive entropy, and so must satisfy (1) and (2). It is quite easy to prove (1) directly from the definition of block gluing, but the corresponding implication of (2) does not seem explicitly known, and so we state it here as a corollary.

Corollary 3.2. For any block gluing $\mathbb{Z}^{d} S F T X$ and any patterns $w, w^{\prime} \in L_{S}(X)$, $w$ and $w^{\prime}$ are chain exchangeable.

Corollary 3.2 is somewhat surprising; recent work has shed light on the fact that there are fundamental differences between uniform mixing conditions involving "surrounding" a pattern (such as uniform filling) and uniform mixing conditions which do not (such as block gluing). (For instance, any SFT $X$ with the UFP cannot have a proper subsystem of entropy $h(X)([7])$, but there are block gluing SFTs which do ([2]).) Though chain exchangeability is quite far from a uniform mixing condition, it is still a condition that involves patterns surrounded by a common border, and so it is a bit unexpected that it would be implied by block gluing. 


\section{ACKNOWLEDGEMENTS}

The author would like to acknowledge the hospitality of the University of British Columbia, where the author was visiting during some of this work.

\section{REFERENCES}

[1] F. Blanchard, Fully positive topological entropy and topological mixing, Symbolic dynamics and applications (in honor of R.L. Adler), AMS Contemporary Mathematics, Providence, RI, 135 (1992), 95-105.

[2] M. Boyle, R. Pavlov and M. Schraudner, Multidimensional sofic shifts without separation, and their factors, Trans. Amer. Math. Soc. 362 (2010), 4617-4653.

[3] M. Boyle and M. Schraudner, $\mathbb{Z}^{d}$ group shifts and Bernoulli factors, Ergodic Theory Dynam. Systems 28 (2008), no. 2, 367-387.

[4] M. Hochman, On the automorphism groups of multidimensional SFTs, Ergodic Theory Dynam. Systems 30 (2010), no. 3, 809-840.

[5] B. Kitchens and K. Schmidt, Automorphisms of compact groups, Ergodic Theory Dynam. Systems 9 (1989), no. 4, 691-735.

[6] D. Lind and B. Marcus, An Introduction to Symbolic Dynamics and Coding, Cambridge University Press, 1995.

[7] A. Quas and A. Sahin, Entropy gaps and locally maximal entropy in $\mathbb{Z}^{d}$ subshifts, Ergodic Theory Dynam. Systems 23 (2003), 1227-1245.

[8] T. Ward, Automorphisms of $\mathbb{Z}^{d}$-subshifts of finite type, Indag. Math. New Ser. 5 (1994), no. 4, 495-504.

Ronnie Pavlov, Department of Mathematics, University of Denver, 2360 S. Gaylord St., Denver, CO 80208

E-mail address: rpavlov@du.edu

URL: www.math.du.edu/ rpavlov/ 